\title{
Fast computation of electromagnetic near-fields with the multilevel fast multipole method combining near-field and far-field translations
}

\author{
A. Tzoulis ${ }^{1}$ and T. F. Eibert ${ }^{2}$ \\ ${ }^{1}$ FGAN-Research Institute for High-Frequency Physics and Radar Techniques (FHR), Wachtberg-Werthhoven, Germany \\ ${ }^{2}$ Institute of Radio Frequency Technology, University of Stuttgart, Germany
}

\begin{abstract}
In Electromagnetic Compatibility (EMC) problems, computation of electromagnetic near-fields in the vicinity of complex radiation and scattering systems is often required. Numerical solution of such problems is achieved using Boundary Integral (BI) based approaches, where the involved Integral Equations (IE's) are solved with the Method of Moments (MoM). The MoM solution process is speeded up by fast IE solvers such as the Multilevel Fast Multipole Method (MLFMM). In the end the desired amplitudes of the expansion of the equivalent current densities on the discrete elements all over the Huygens' surfaces are known. Computation of the electromagnetic fields produced by the equivalent currents at observation points being in the near-field regions requires integration of the current densities over the Huygens' surfaces. Numerical evaluation of the near-field integrals using conventional integration rules can become extremely time consuming for large objects and large number of observation points. In this contribution, acceleration of the near-field integration of the equivalent current densities is provided using a postprocessing MLFMM, where near-field and far-field translations are combined in order to achieve optimum performance. The proposed approach was applied in the postprocessing stage of a powerful Finite Element Boundary Element (FEBI) method, resulting in significant decrease of the postprocessing computation time. The formulation of the proposed acceleration is presented and numerical results are shown.
\end{abstract}

\section{Introduction}

Complex radiation and scattering problems are solved these days routinely using Boundary Integral (BI) based methods, where the Integral Equation's (IE's) are solved by the Method of Moments (MoM) (Rao et al., 1982) using fast integral

Correspondence to: A. Tzoulis (tzoulis@ fgan.de) equation solvers like the Multilevel Fast Multipole Method (MLFMM) (Chew et al., 2001; Eibert, 2005). The MoM solution provides the desired amplitudes of the expansion of the equivalent current densities on the discrete elements all over the Huygens' surfaces.

In order to compute the electromagnetic fields produced by the equivalent currents on observation points in the near-field region of the involved objects, integration of the current densities over the entire Huygens' surfaces must be performed in the postprocessing stage. Such computations are needed in Electromagnetic Compatibility (EMC) problems like investigations within cars or for safety assessment in the vicinity of mobile communications base station antennas. The complexity of evaluating near-field integrals in the postprocessing stage using conventional numerical integration rules is $O(N M)$, where $N$ is the number of equivalent current elements and $M$ the number of observation points. It is obvious that postprocessing computations become extremely time-consuming in case of large objects and large number of observation points.

In this contribution, near-field computations in the postprocessing stage are accelerated by a Postprocessing Multilevel Fast Multipole Method (P-MLFMM). According to this, the BI-MLFMM domain of the equivalent currents is extended to also include nearby observation points and in addition to non-empty groups containing source currents also non-empty groups containing observation points are collected on each level. Near-field MLFMM translations are performed from source groups including currents to receiving groups including observation points. For far-away observation points, the field contributions are computed using far-field MLFMM translations, which are performed for each observation point on the coarsest level on which far-field condition is still satisfied. The optimum level for far-field translations is found for each observation point in the initialization step in a worst-case sense, using its shortest distance to the BI-MLFMM domain. The proposed approach was ap-

Published by Copernicus GmbH on behalf of the URSI Landesausschuss in der Bundesrepublik Deutschland e.V. 


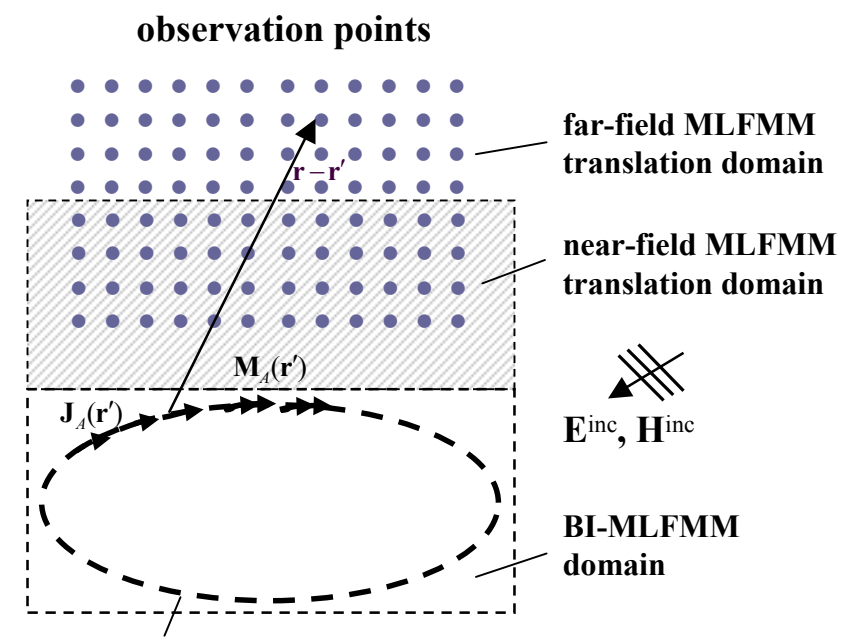

closed surface $A$

Fig. 1. Configuration for postprocessing computations of BI based methods.

plied in the postprocessing stage of a powerful Finite Element Boundary Element (FEBI) method (Jin, 1993; Volakis et al., 1998), resulting in significant decrease of the postprocessing computation time.

In the following, the formulation of the MLFMM approach for the postprocessing stage is presented and numerical results are shown.

\section{Formulation}

Consider the configuration of Fig. 1, where electric $\boldsymbol{J}_{A}\left(\boldsymbol{r}^{\prime}\right)$ and magnetic $\boldsymbol{M}_{A}\left(\boldsymbol{r}^{\prime}\right)$ current densities are given all over the closed Huygens' surface $A$ and are known from the solution of any BI based method.

The electric and magnetic fields at an observation point $\boldsymbol{r}$ produced by the surface currents are given by the integral expressions

$$
\begin{aligned}
\boldsymbol{E}(\boldsymbol{r})= & \iiint_{A}\left[\overline{\mathbf{G}}_{J}^{E}\left(\boldsymbol{r}, \boldsymbol{r}^{\prime}\right) \cdot \boldsymbol{J}_{A}\left(\boldsymbol{r}^{\prime}\right)+\overline{\mathbf{G}}_{M}^{E}\left(\boldsymbol{r}, \boldsymbol{r}^{\prime}\right)\right. \\
& \left.\cdot \boldsymbol{M}_{A}\left(\boldsymbol{r}^{\prime}\right)\right] d a^{\prime}+\boldsymbol{E}^{i n c}(\boldsymbol{r})
\end{aligned}
$$

and

$$
\begin{aligned}
\boldsymbol{H}(\boldsymbol{r})= & \iiint_{A}\left[\overline{\mathbf{G}}_{J}^{H}\left(\boldsymbol{r}, \boldsymbol{r}^{\prime}\right) \cdot \boldsymbol{J}_{A}\left(\boldsymbol{r}^{\prime}\right)+\overline{\mathbf{G}}_{M}^{H}\left(\boldsymbol{r}, \boldsymbol{r}^{\prime}\right)\right. \\
& \left.\cdot \boldsymbol{M}_{A}\left(\boldsymbol{r}^{\prime}\right)\right] d a^{\prime}+\boldsymbol{H}^{i n c}(\boldsymbol{r}),
\end{aligned}
$$

respectively, where $\overline{\mathbf{G}}_{J}^{E / H}\left(\boldsymbol{r}, \boldsymbol{r}^{\prime}\right)$ and $\overline{\mathbf{G}}_{M}^{E / H}\left(\boldsymbol{r}, \boldsymbol{r}^{\prime}\right)$ are the Green's functions of the electric or magnetic field due to electric and magnetic surface currents, respectively. The equivalent currents on $A$ are assumed to be available in a known expansion using RWG basis functions on triangular surface elements according to

$$
\begin{aligned}
\boldsymbol{J}_{A}\left(\boldsymbol{r}^{\prime}\right) & =\sum_{n} J_{n} \beta_{n}\left(\boldsymbol{r}_{\boldsymbol{n}}\right), \\
\boldsymbol{M}_{A}\left(\boldsymbol{r}^{\prime}\right) & =-\sum_{n} M_{n} \beta_{n}\left(\boldsymbol{r}_{\boldsymbol{n}}\right) .
\end{aligned}
$$

The evaluation of the field contributions in Eqs. (1) and (2) is accelerated using MLFMM. According to this, the BIMLFMM domain is extended towards the observation points and in addition to non-empty source groups containing currents also non-empty receiving groups containing observation points are collected on each level. The field contributions from source groups to non well-separated receiving groups are evaluated conventionally by numerical integration rules. All other field contributions at an observation point $r=r_{m}$ are computed using the fast multipole representations

$$
\begin{gathered}
\boldsymbol{E}\left(\boldsymbol{r}_{\boldsymbol{m}}\right)=-j \frac{\omega \mu}{4 \pi} \oiint e^{-j \boldsymbol{k} \cdot \boldsymbol{r}_{m m^{\prime}}} T_{L}\left(\hat{k} \cdot \hat{r}_{m^{\prime} n^{\prime}}\right)(\overline{\mathbf{I}}-\hat{k} \hat{k}) \\
\cdot \sum_{n} J_{n} \tilde{\beta}_{n}(\hat{k}) d \hat{k}^{2} \\
-j \frac{k}{4 \pi} \oiint_{\hat{k} \times e^{-j \boldsymbol{k} \cdot \boldsymbol{r}_{m m^{\prime}}} T_{L}\left(\hat{k} \cdot \hat{r}_{m^{\prime} n^{\prime}}\right)} \\
\sum_{n} M_{n} \tilde{\beta}_{n}(\hat{k}) d \hat{k}^{2}+\boldsymbol{E}^{i n c}\left(\boldsymbol{r}_{\boldsymbol{m}}\right)
\end{gathered}
$$

and

$$
\begin{aligned}
& \boldsymbol{H}\left(\boldsymbol{r}_{\boldsymbol{m}}\right)=-j \frac{k}{4 \pi} \oiint \hat{k} \times e^{-j \boldsymbol{k} \cdot \boldsymbol{r}_{m m^{\prime}}} T_{L}\left(\hat{k} \cdot \hat{r}_{m^{\prime} n^{\prime}}\right) \\
& \sum_{n} \tilde{J}_{n} \tilde{\beta}_{n}(\hat{k}) d \hat{k}^{2} \\
&+j \frac{\omega \varepsilon}{4 \pi} \oiint^{-j \boldsymbol{k} \cdot \boldsymbol{r}_{m m^{\prime}}} T_{L}\left(\hat{k} \cdot \hat{r}_{m^{\prime} n^{\prime}}\right)(\overline{\mathbf{I}}-\hat{k} \hat{k}) \\
& \cdot \sum_{n} M_{n} \tilde{\beta}_{n}(\hat{k}) d \hat{k}^{2}+\boldsymbol{H}^{i n c}\left(\boldsymbol{r}_{\boldsymbol{m}}\right),
\end{aligned}
$$

where

$$
\tilde{\beta}_{n}(\hat{k})=\iint_{A} \beta_{n}\left(r_{n}\right) e^{j k \cdot r_{n n^{\prime}}} d a_{n}
$$

is the $\hat{k}$-space representation of the source basis functions with $\boldsymbol{k}=k \hat{k} . T_{L}\left(\hat{k} \cdot \hat{r}_{m^{\prime} n^{\prime}}\right)$ is the conventional FMM diagonal translation operator (Chew et al., 2001). This procedure is here referred to as near-field MLFMM translation, by means of plane wave representation of the radiated fields of source groups containing currents with integration over the Ewald sphere and translation of the plane waves to received field contributions at groups containing observation points. The radiation patterns of the source groups are computed in the center points using the spherical harmonics expansion of the current basis functions according to Eibert (2005). 


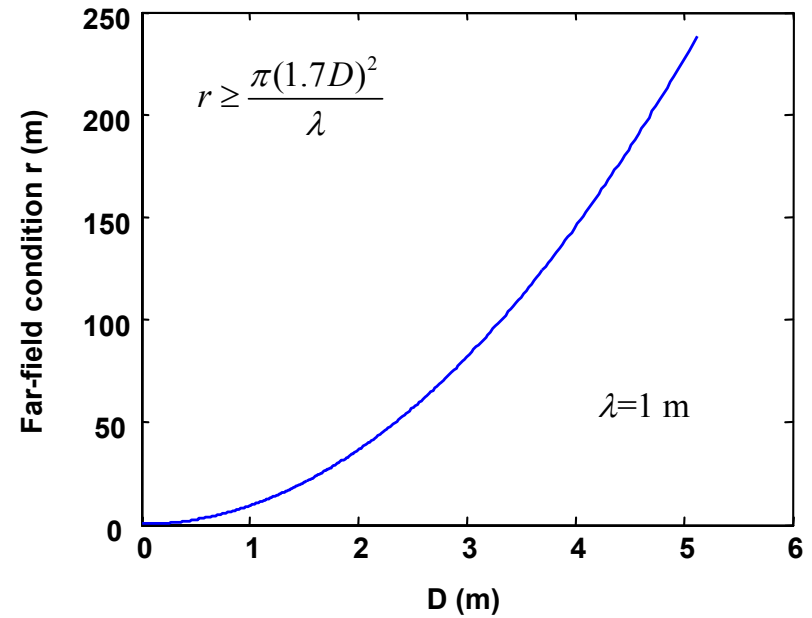

Fig. 2. Far-field condition with increasing group dimensions.

Extending the near-field translation domain to all observation points can be extremely memory-consuming. Therefore, near-field MLFMM translations are applied only to nearby observation points and far-away observation points are preferably treated by far-field MLFMM translations, where the translation operator is approximated by a far-field representation only for the ray direction from the source group to the observation point. Thereby, it is assumed that the observation point lies in the far-field of the individual source group. The field contributions for an observation point $r=r_{m}$ within the far-field MLFMM translation domain are given by

$$
\begin{aligned}
\boldsymbol{E}\left(\boldsymbol{r}_{\boldsymbol{m}}\right)= & -j \frac{\omega \mu}{4 \pi} T_{L}^{F F}\left(k r_{m n^{\prime}}\right)\left(\overline{\mathbf{I}}-\hat{k}_{0} \hat{k}_{0}\right) \cdot \sum_{n} J_{n} \tilde{\beta}_{n}\left(\hat{k}_{0}\right) \\
& -j \frac{k}{4 \pi} \hat{k}_{0} \times T_{L}^{F F}\left(k r_{m n^{\prime}}\right) \sum_{n} M_{n} \tilde{\beta}_{n}\left(\hat{k}_{0}\right) \\
& +\boldsymbol{E}^{i n c}\left(\boldsymbol{r}_{\boldsymbol{m}}\right)
\end{aligned}
$$

and

$$
\begin{aligned}
\boldsymbol{H}\left(\boldsymbol{r}_{\boldsymbol{m}}\right)= & -j \frac{k}{4 \pi} \hat{k}_{0} \times T_{L}^{F F}\left(k r_{m n^{\prime}}\right) \sum_{n} J_{n} \tilde{\beta}_{n}\left(\hat{k}_{0}\right) \\
& +j \frac{\omega \varepsilon}{4 \pi} T_{L}^{F F}\left(k r_{m n^{\prime}}\right)\left(\overline{\mathbf{I}}-\hat{k}_{0} \hat{k}_{0}\right) \cdot \sum_{n} M_{n} \tilde{\beta}_{n}\left(\hat{k}_{0}\right) \\
& +\boldsymbol{H}^{i n c}\left(\boldsymbol{r}_{\boldsymbol{m}}\right)
\end{aligned}
$$

where $T_{L}^{F F}\left(k r_{m n^{\prime}}\right)$ is the far-field MLFMM translation operator in the ray direction $\hat{k}_{0}=\hat{r}_{m n^{\prime}}$ (Chew et al., 2002). Improved far-field representation is obtained using the gravity center of the currents within the source groups as reference point for the radiated fields of the groups. The necessary ray direction $\hat{k}_{0}$, which connects the gravity center of the currents within the source group with the observation point, is computed from the neighboring sampling points of the numerical integration in the $\hat{k}$-space by interpolation.
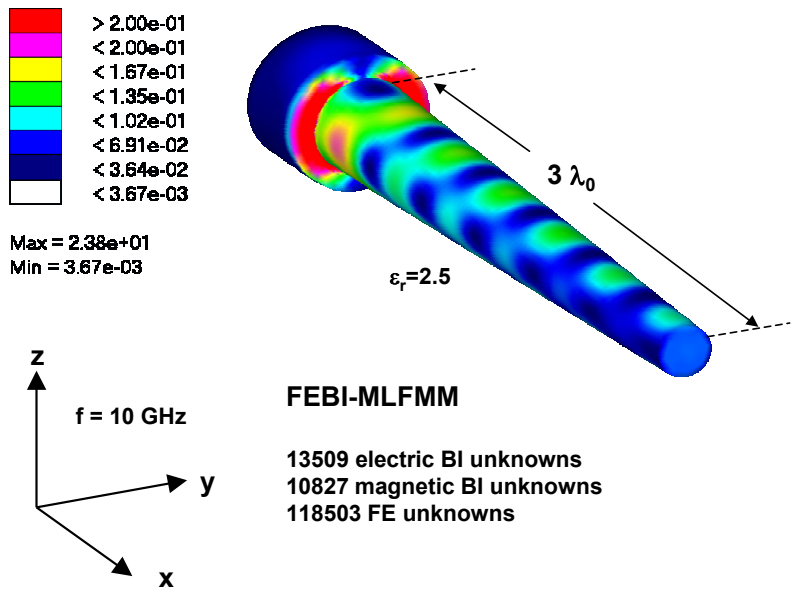

FEBI-MLFMM

13509 electric $B I$ unknowns 10827 magnetic $B I$ unknowns $118503 \mathrm{FE}$ unknowns

Fig. 3. Real part of surface current density on dielectric rod antenna.

The condition used to perform far-field translations is

$r_{f a r} \geq \frac{\pi\left(1.7 D_{L}\right)^{2}}{\lambda}$

and is derived from the criterion for far-field translations according to the Fast Far-Field Approximation (FAFFA) in (Chew et al., 2002). $D_{L}$ is the group dimension on level $L$ and $\lambda$ is the wavelength. The condition increases with the square of group dimensions, as shown in Fig. 2, which means that enforcing satisfaction of this condition for the whole scatterer or antenna would be very inefficient. It is rather assumed that far-field condition is satisfied by the individual source groups on the various levels. For each observation point, far-field translations are performed on the coarsest level $L$, on which condition (9) is still satisfied. This level is found for each observation point in the initialization step in a worst-case sense using its shortest distance to the BIMLFMM domain. Improved accuracy is provided by choosing a more stringent far-field condition.

The proposed acceleration of near-field computations was applied at the postprocessing stage of a powerful FEBI method. In the next section numerical results showing the savings in computation time will be presented.

\section{Numerical examples}

First numerical example is the dielectric rod antenna shown in Fig. 3. The operating frequency of the antenna was 10 $\mathrm{GHz}$ and a delta-gap voltage source was used for excitation. In the same figure, the real part of the equivalent surface current density derived from the FEBI solution can be seen, using a tetrahedral FE volume mesh with 118503 unknowns and a triangular surface mesh with 13509 electric and 10827 magnetic unknowns. In the postprocessing stage the electric field was first observed on 6901 observation points along the antenna axis, as shown in Fig. 4. The computations were performed with the postprocessing MLFMM approach and with 


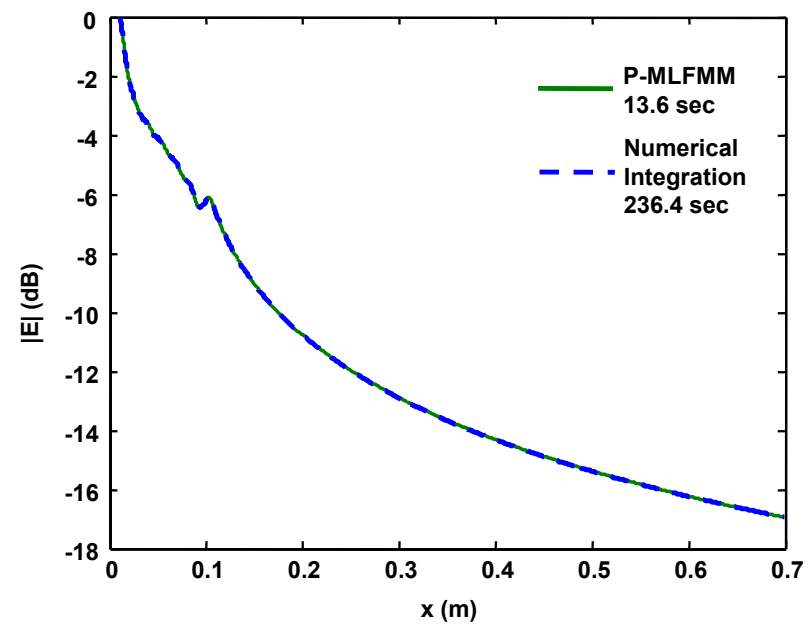

Fig. 4. Electric field along the axis of dielectric rod antenna.

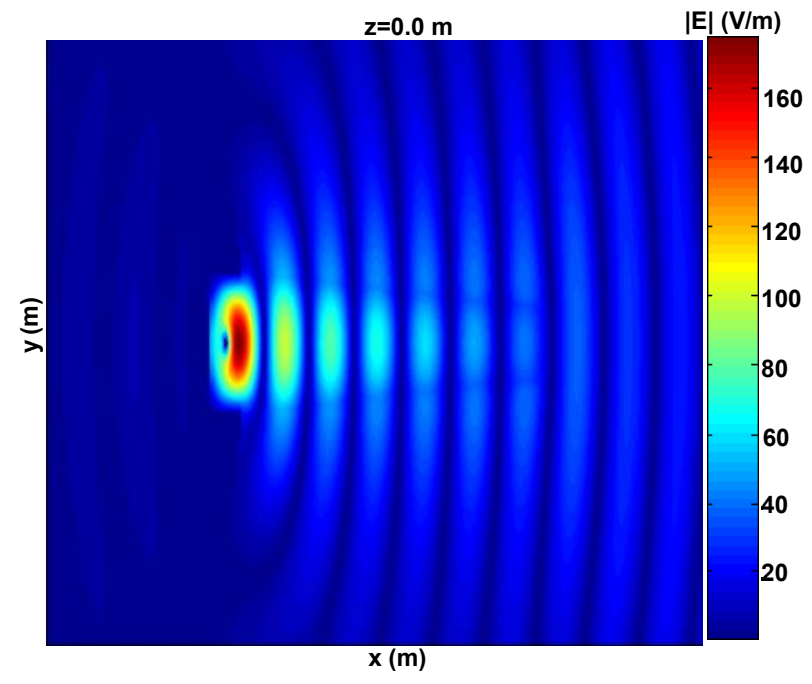

Fig. 5. Instantaneous electric field for $t=0 \mathrm{~s}$ on the xy-plane.

conventional near-field integration. It can be seen that results show excellent agreement and the P-MLFMM computations need much less computation time. Further, the instantaneous electric field for $t=0 \mathrm{~s}$ can be seen in Fig. 5 on the xy-plane, cutting through the antenna in the middle along its axis. These computations were performed on 100701 observation points with the P-MLFMM approach in about $7 \mathrm{~min}$ on an AMD Athlon 2800+ PC combining near-field and farfield translations. The wave radiated by the antenna mainly at the transition region between metallic mounting and dielectric material with very low radiation in the backward direction can be clearly seen.

Next example is the open metallic cuboid shown in Fig. 6. The length of the structure is $30 \lambda$ and the square cross section has an inner edge length of $10 \lambda$. The object is placed symmetrically to the xy-plane. In the same figure the magnitude of the surface current density on the metallic cuboid

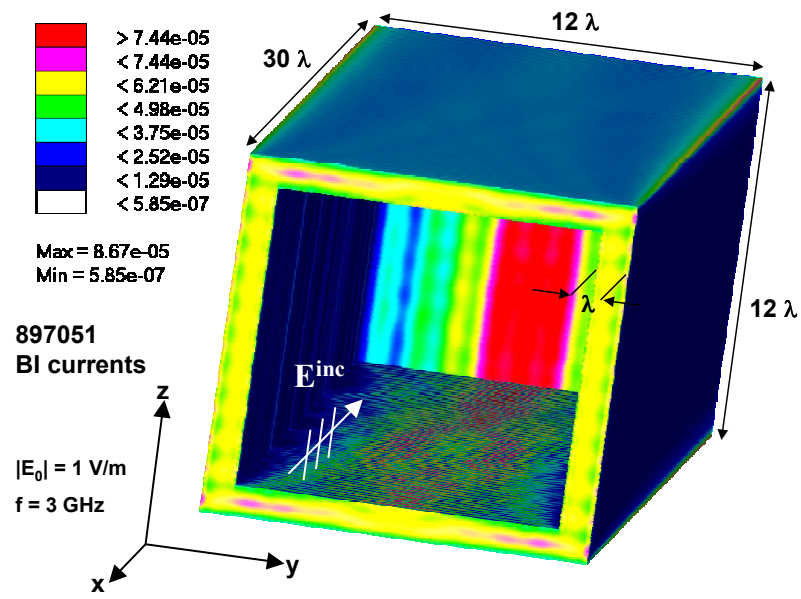

Fig. 6. Magnitude of surface current density on open metallic $30 \lambda$ cuboid.

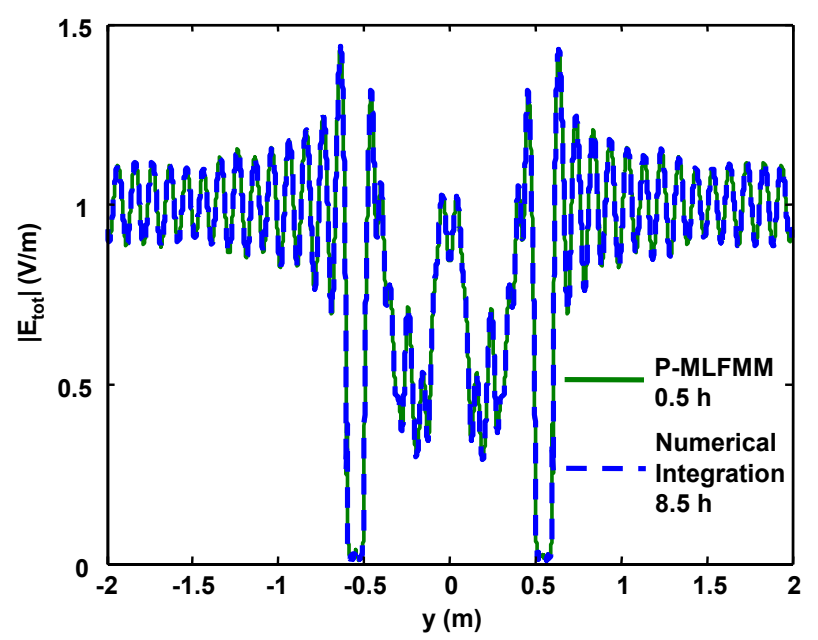

Fig. 7. Total electric field along the y-axis for open metallic cuboid.

can be seen, which is the BI solution of 897051 surface currents for vertically polarized plane wave excitation traveling towards the opening of the cuboid. In the postprocessing stage, the electric field was observed along the y-axis on 10001 observation points by performing the computations with the fast postprocessing approach and with conventional near-field integration. The results are shown in Fig. 7, where excellent agreement and saving of large amount of computation time can be seen. Further, the electric field was observed on a $2 \times 4 \mathrm{~m}^{2}$ window in the xy-plane with 1 mill. observation points. The computations were performed with the $\mathrm{P}$ MLFMM approach in about $8 \mathrm{~h}$ combining near-field and farfield translations. The estimated computation time with conventional numerical integration would be about $850 \mathrm{~h}$, which is unacceptably high. The results are shown in Fig. 8, where the expected distribution of the electric field inside, outside and within the metallic walls of the cuboid can be seen.

Last example is the full-scale model of a Mig-29 shown 


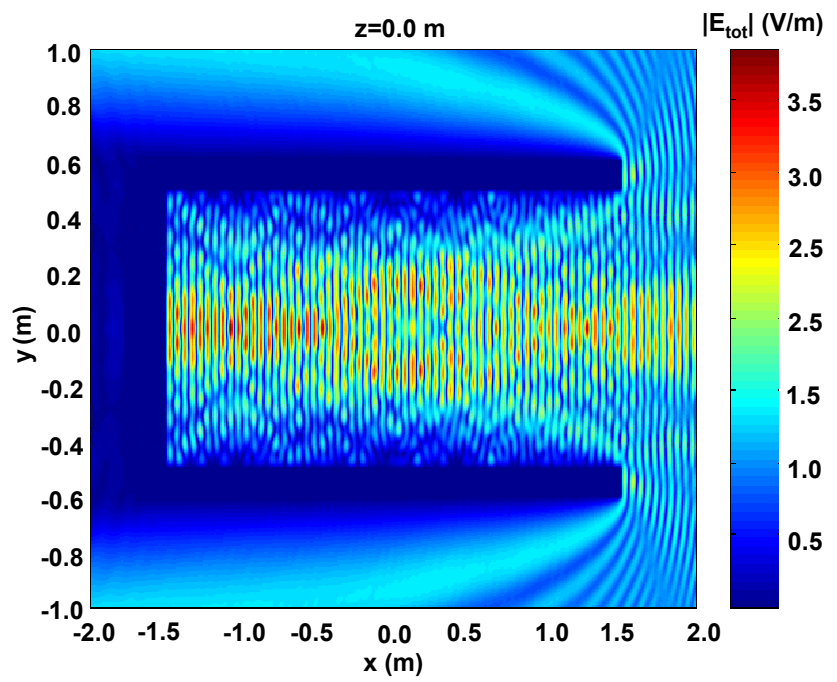

Fig. 8. Total electric field through the open metallic cuboid.
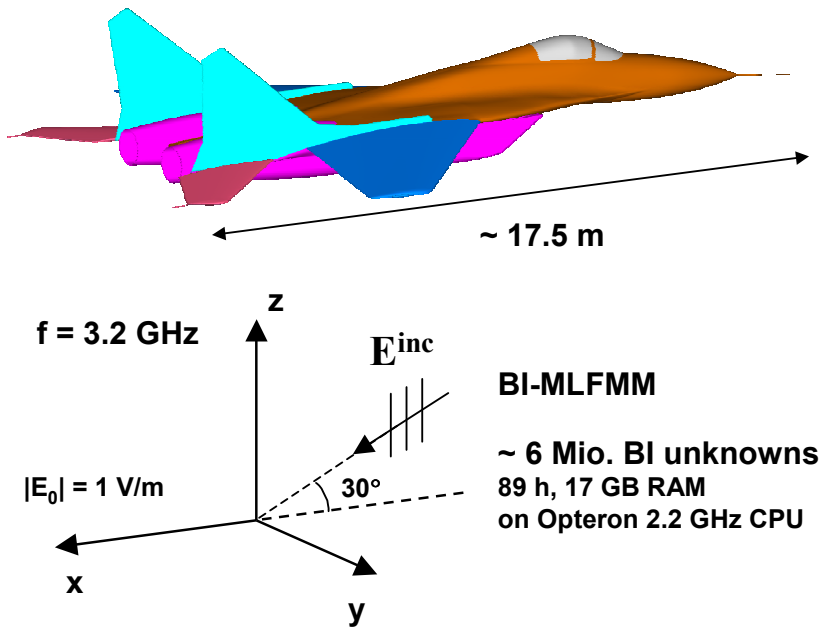

Fig. 9. Full-scale Mig-29 model for $f=3.2 \mathrm{GHz}$.

in Fig. 9. The model is about $17.5 \mathrm{~m}$ long and was excited by a vertically polarized plane wave traveling $150^{\circ}$ from the $\mathrm{x}$-axis towards the nose of the aircraft. The BI solution of this problem was performed on an AMD Opteron $2.2 \mathrm{GHz}$ CPU using about 6 mill. unknowns. In the postprocessing stage the near-field was observed through the aircraft on a plane parallel to the xy-plane on about 2.2 mill. observation points. These computations were performed with the P-MLFMM approach in about $3 \mathrm{~h}$, combining near-field and far-field translations. The magnitude of the total electric near-field through the Mig-29 is shown in Fig. 10, where the expected distribution of the electric field can be seen.

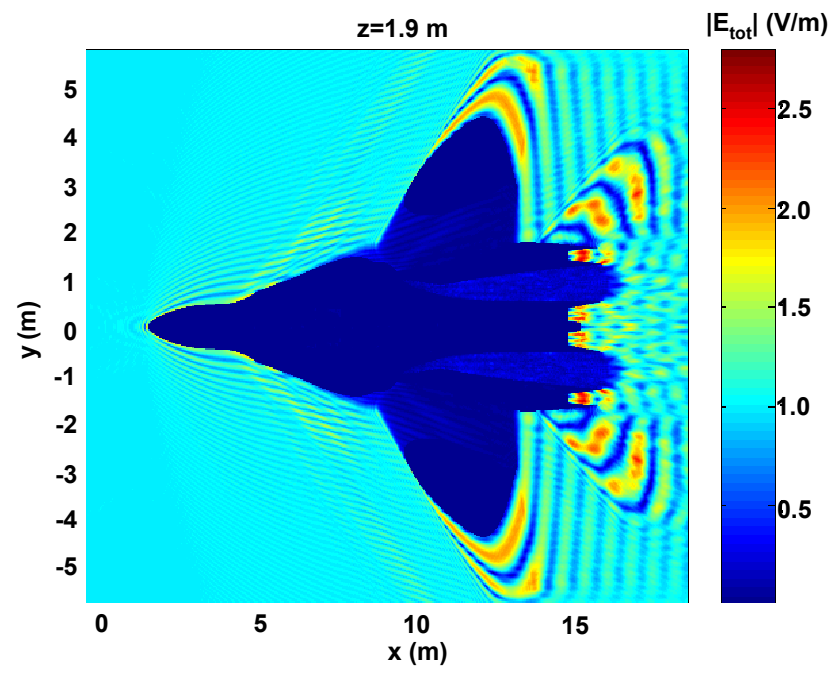

P-MLFMM on 2.2 Mio observation points $3 \mathrm{~h}, 8 \mathrm{~GB}$ RAM on Opteron $2.2 \mathrm{GHz} \mathrm{CPU}$

Fig. 10. Total electric field through Mig-29.

\section{Conclusions}

In this contribution, the multilevel fast multipole method was used to accelerate near-field computations in the postprocessing stage of boundary integral based methods for numerical solution of electromagnetic radiation and scattering problems, where optimum performance is achieved combining near-field and far-field translations. The proposed PMLFMM was applied in the postprocessing stage of a powerful FEBI method and numerical results were shown, where saving of large amount of computation time in the postprocessing stage could be clearly seen.

\section{References}

Chew, W. C., Jin, J.-M., and Michielssen, E.: Fast and efficient algorithms in computational electromagnetics, Boston: Artech House, 2001.

Chew, W. C., Cui, T. J., and Song, J. M.: A FAFFA-MLFMA algorithm for electromagnetic scattering, IEEE Trans. Antennas Propagat., 50, 1641-1649, 2002.

Eibert, T. F.: A Diagonalized Multilevel Fast Multipole Method with Spherical Harmonics Expansion of the $k$-Space Integrals, IEEE Trans. Antennas Propagat., 53, 814-817, 2005.

Jin, J.-M.: The Finite Element Method in Electromagnetics, 2nd Ed., John Wiley and Sons, Inc., New York, 2002.

Rao, S. M., Wilton, D. R., and Glisson, A. W.: Electromagnetic scattering by surfaces of arbitrary shape, IEEE Trans. AP, 30, 409-418, 1982.

Volakis, J. L., Chatterjee, A., and Kempel, L.-C.: Finite Element Method for Electromagnetics, IEEE, Inc., New York, 1998. 